\title{
Assessing Towards the Perception of Physical Activities of People in Prevention of Non-communicable Diseases in the Case of Debre Berhan and Showa Robit Towns
}

\author{
Arefayne Mesfen, Abiot Kassaw, Belay Lema, Birhanu Anjte \\ Department of Sport Science, College of Natural and Computational Sciences, Debre Birhan University, Debre Birhan, Ethiopia \\ Email address: \\ Mesfendessye@gmai.com (A. Mesfen)
}

\section{To cite this article:}

Arefayne Mesfen, Abiot Kassaw, Belay Lema, Birhanu Anjte. Assessing Towards the Perception of Physical Activities of People in Prevention of Non-communicable Diseases in the Case of Debre Berhan and Showa Robit Towns. American Journal of Sports Science. Vol. 4, No. 4, 2016, pp. 61-68. doi: 10.11648/j.ajss.20160404.11

Received: November 11, 2015; Accepted: November 25, 2015; Published: June 7, 2016

\begin{abstract}
This study atte mpted to assess the habits and awareness of people towards physical exercise, its challenges and opportunities in the process of preventing selected NCDs. To conduct this, the researchers delimited to Debre Berhan and Showa Robit towns in different respective population groups and offices. The total sample sizes were 3200 and a minimum of 900 sample respondents were taken by $10 \%$ rule among this 743 for questionnaire and 157 for interview respondents. Each office has been selected by stratified sampling based on purposively expected availability of information and participants categorized according to their demographic placement such as sex, age, education and habit of involvement then followed by simple random sampling techniques. The data collection tools employed questionnaires, interview with focus group discussion and document were analyzed in triangulation manner. The reason why the researchers chooses these methods to get valuable and reliable data for numerous barriers of physical exercise, prevention disease (NCDs) in the case of Debre Birhan and Showa Robit town. Then the questionnaires were administered and the data was collected, tailored, analyzed and logical conclusion and recommendation was reported.
\end{abstract}

Keywords: Perception, Physical Exercise, Non-communicable Diseases

\section{Introduction}

The findings of different literature display that throughout the world the majority of the community health affected by non-communicable diseases NCDs- (silent killers). As the data shown our country is part of this global and African society from the world experience as reported the results of NCDs every year in the minimum of: 2.8 million People death occurred by high obesity (cholesterol) 4.4 million People death occurred by high blood pressure. As WHO point out in his report physical inactivity is one of the leading dangerous cause for 7.2 million people death occurred annually. (WHO workshop on physical activity and public Health 24-27october 2005, in Beijing people, Republic of China)

Extensive research clearly states that the adoption and maintenance of moderate levels of physical activity provides tremendous benefits for physical and mental health.
Chronic non-communicable diseases are largely due to preventable and modifiable risk factors such as, high blood cholesterol, high blood pressure, obesity, physical inactivity, unhealthy diet, tobacco use and inappropriate use of alcohol. These factors result in various long-term disease processes, culminating in high mortality rates attributable to stroke, heart attack, tobacco- and nutrition-induced cancers, obstructive lung diseases and many others. (Thandi Puoane and et. al)

\subsection{People Participate in Physical Exercise}

1. Barriers of local government recreation departments (lack of nearer support)

2. Barriers of processes and structures (silence in the process of decision)

3. Barriers of programming and infrastructure (rigidity of programs related to facilities)

4. Barriers of economics (unaffordable of physical activity costs) 


\section{Barriers of transportation}

6. Barriers of social and physical skills

7. Barriers of societal attitudes

8. Barriers of individual and family perceptions, attitudes and knowledge

9. Barriers for cultural and visible minorities

10. Barriers in small, rural and remote communities

People affected by poverty may have fewer opportunities to build the skills they need to participate. If they didn't participate when they were children, people may lack the social and team skills, and physical ability to participate at the same level as others. (Tara Spruce Stimson)

\subsection{Theoretical Models and the Adoption of Physical Activity}

School of thoughts or theoretically synthesized from: Tran's theoretical model (behavioral change), decisionmaking theory, and social cognitive theory (to specific selfefficacy) strategies theory have been used to gain insight on how individuals decide to become physically active. (Tara Spruce Stimson)

\subsubsection{Trans-Theoretical Model}

The trans-theoretical model, commonly recognized as the stages of change model, has been successfully applied to tailoring interventions. This model has been effectively used in tailoring treatment to not interested individuals and engaging in physical activity and has been useful in guiding and developing successful physical activity interventions. Increasingly, researchers are using the trans-theoretical model of behavior change as a theoretical framework for examining the exercise adoption and maintenance process. The stages of change model proposed by Prochaska and DiClimente describe the different phases involved in the acquisition and maintenance of behavior. These researchers suggest that individuals engaging in a new behavior and move an orderly progression through five different stages:

1. Pre-contemplation (no intention to change behavior)

2. Contemplation (intention to change behavior)

3. Preparation (making small changes)

4. Action (involved in behavior change)

5. Maintenance (sustained behavior change)

A particular strength in applying the stages of change model to the study of physical activity behaviors is its dynamic nature. The model focuses on the transitions that occur in adoption and maintenance of a behavior, with individuals progressing through the stages at varying rates. Movement through the stages does not always occur in a linear manner, but may also be cyclical as most individuals make several attempts at behavior change before the goal is met.

Project Active was a two-year randomized trial that compared lifestyle physical activity with a structured exercise program. Both groups at six months became significantly more active. The materials were developed to specifically target cognitive and behavior stages of change based on Prochaska and DiClimente stages of change model and Bandura's 18 cognitive theory that analyzed selfefficacy. Results indicate that change during the first six months predicted physical activity behavior at 24 months. (Tara Spruce Stimson)

\subsubsection{Decisional Balance Theory}

Decision making theories are an important component in understanding behavior change. Studies of decisional balance measure, the individual's evaluation of the pros and cons related to physical activity behavior. Results have shown an association between a person's relative view of pros, cons and readiness for physical activity adoption.

Prochaska et al. have concluded that the original eight central constructs developed by Janis and Mann could be reduced to a simpler two-factor model consisting of Pros (benefits) and Cons (barriers). Pros represent the perceived benefits of behavior change, whereas, the cons represent the perceived barriers to behavior change. Prochaska et al. maintain that pros and cons are excellent indicators of an individual's progress from pre-contemplation to contemplation and preparation stages.

\subsubsection{Self-Efficacy Theory}

Investigations applying the stages of change model to exercise behavior have also focused on the construct of exercise self-efficacy. Self-efficacy is the degree of confidence that an individual has in his/her ability to be physically active in specific situations. Self-efficacy has been shown to be positively associated with the adoption and maintenance of physical activity behavior and motivational readiness for physical activity participation. Bandura maintains that individuals with high confidence in their ability to perform a given task will be more likely to engage in that task. Research using the trans-theoretical model has found that self-efficacy scores correlate highly with stages of change. In the pre-contemplation stage, self-efficacy scores have a tendency to be lower than in the maintenance stage. (Tara Spruce Stimson)

\subsection{Investments in Physical Activity}

- The health aspects of physical activity

- The social benefits of physical activity

- The economic benefit or cost savings of physical activity

\subsection{Statement of the Problem}

As the data illustrates above globally many people have challenged by non-communicable diseases, the scarcity of this regular physical activity affects:

Health- affects both physiological, psychological and social aspects of health such as increase coronary heart disease, stroke, diabetes, site specific cancers (colon, breast and lung cancer), osteoporosis, obesity, increase (stress, anxiety and depression). Economy- this alliance loss productivity labor, physically inactive people estimated \$211 million costs annually for health care's and socialization (the interaction of people) affected by physical inactivity. 
Stated by (Christine Hancock, 2011)

Therefore the aforementioned problem was observed in Debre Berhan and Showa Robit town as the data obtained from health centers, reference (Debre Berhan referral hospital and private health station). On the reverse physical exercise is not that much observed. Due to this logical reasoning the researchers were dig out the following issues:

Basic research questions

- To what extent the community is aware about the importance of physical exercise related to health, economy and social benefit?

- What are the major factors affect the participation of the community towards regular physical activities?

- Are there sport arena and fitness centers available in Debre Birhan and Showa Robit town?

- To what extent the community involving in physical exercise in the available sport arena and fitness center to combat NCDS alliance?

For theses issue, the study alleviated truly the aforementioned issues entirely in the organ of the research.

\subsection{Objectives of the Study}

\subsubsection{General Objective}

- This research was tried to assess the perception, habits and awareness of people towards physical exercise and its challenges and opportunities in the process of preventing selected NCDs.

\subsubsection{Specific Objectives}

- To examine the community perception towards the importance of physical exercise in order to prevent NCDs

- To assess the habits of sport practice and awareness of the community towards the benefit of physical exercise

- To assess the major hindering barriers of the community towards the involvement of regular physical exercise

- To identify the current status of sport arena and fitness centers

- To add value for the country by keeping the community health prevented from non-communicable disease.

\subsection{Significance of the Study}

This study were conducted to identify the major challenges in the practice of physical fitness in Debre Birhan and Showa Robit town community alienated to prevent selected NCDs chronic health conditions. Therefore the researchers hopes that the findings of the research would contribute to: Dig out and display the major barriers of the people habits and sport culture, the importance of exercise to society active life style, healthy and health related issues are timely researchable area since especially the developing world (low income country people) have not obtained sufficient medical services because the diagnosis and treatment costs are very expensive, to solve this problem enhancing the practice of physical exercise became the preferable means and no choice, to give insight for experts within the same streams; and also to provide scientific findings for policy makers in behalf of prevention program.

\section{Methodology}

\subsection{The Study Area}

The study was to assess the perception, habits and awareness of people towards the effects of physical exercise and its challenges and opportunities in the process of preventing selected NCDs in Debre Berhan and Showa Robit towns' community in the form of descriptive survey.

\subsection{Research Design}

The study was employed descriptive research design which is the road map it refers to a plan of action that links the philosophical assumption to specific methods (Kumar, 1999). It is important in order to provide sufficient information regarding the subject, it saves time and again the study focused on deeply with associated factors harmony to environment like lifestyle, lack of time, social factors, habits of sport practice, culture of sport ethics, lack of interest, fear of injury, lack of skill, lack of resource (human, facilities and equipment's all in all sport infrastructure alienated to urban plan, transportation with work and residence. The following questionnaire and interview question had been necessary designed to handle all items and alternatives with simple frequency percentage, and were treated entirely based on the study objectives and our basic research questions. Therefore first the questionnaires of the respondents respond by Amharic as much as possible, it is managed and translated in to English genuinely and accordingly the major hindering barriers.

\subsection{Subject of the Study Population (Sample Determination)}

The participants of the study would be delimited in Debre Birhan and Showa Robit town selected sector workers a minimum of $10 \%$ rule the proportion of the total sample size $(\mathrm{N}=3200)$, among this we were taken $(\mathrm{n}=900)$ sample respondents first this were categorized based on sex 743 (501 male and 242 female) respondents for questionnaires, and 157 (97 male and 60 female) for interview, both in Debre Birhan and Showa Robit town separately N1-Debre Birhan=2200, N2-Showa Robit=1000, therefore the proportional formula for the questionnaire and interview is represented by $\mathrm{P}=\mathrm{Ni} \mathrm{X} \mathrm{n} \quad \mathrm{N}, \quad \mathrm{i}=1, \quad 2$ : $\mathrm{P} 1=\mathrm{N} 1 \mathrm{Xn} / \mathrm{N}=2200 \mathrm{X} 900 / 3200=619$ and $\mathrm{P} 2=\mathrm{N} 2 \quad \mathrm{X} \quad \mathrm{n} / \mathrm{N}$ $=1000 X 900 / 3200=281$. Second from 900 sample population were categorized based on places 794 for questionnaire and interview (660 for questionnaire and 134 for interview from Debre Birhan), 106 for questionnaire and interview (83 for questionnaire and 23 for interview) were taken from Showa Robit town purposively.

\subsection{Source of Data}

Primary and secondary data sources were employed

\subsection{Method of Data Analysis}

In this study, both qualitative and quantitative mixed 
analytical research method were employed, for reference to this, Flick (2002) as quieted Muluken (2006) suggested that both were corresponding rather than contestant camps. The researchers offered the questionnaires checked by statisticians, as pilot test first for data validity and reliability and the questionnaires would be distributed accordance with other tools such as prepared interview, focus group discussion, observation and gathering relevant documents from various sources. The techniques of the proper handling system of the collected data were employed by stratum purposively first and utilized simple random sampling and then the responses tabulated and analyzed statistically with simple frequency percentage.

\subsection{Data Collection Instruments (Tools)}

In this research, the data collection tools employed questionnaires, interview mixed with focus group discussion and document were analyzed in triangulation manner. The reason why the researcher chooses these instruments was to get valuable and reliable data for outshined barriers of physical exercise and causes of prevention diseases (NCDs) in the case of Debre Birhan and Showa Robit town.

\subsubsection{Questionnaire}

A questionnaire is one of a data collection tool, in which written questions presented that were to be answered by the respondents in both close ended and open ended question with likert scale form, because it was the most commonly used tools to evaluate closed opinions, therefore the respondents were responding their answer with a pre sheet on the given space accordingly.

\subsubsection{Interview}

Interviews were a type of survey tools where questions were conducted in face to face or personal contact situation of two parties the interviewee and interviewer. The interview is like a conversion and has the purpose of obtaining information relevant to a particular research theme (Kumar, 1999). The researcher's technique would be employed focus group discussion mixed with semi- structured interview (the purpose of this semi- structured interview is to give the opportunity for the free flow of information between interviewer and interviewee).

\subsubsection{Document Analysis}

Documents were obtained from valuable primary and secondary sources of information such as texts, newspaper, minutes of meetings, articles, letters, diaries, memos (scripts) these were manual and electronics obtained from clients and health centers.

\section{Result and Discussion}

Table 1. Description about attitudinal barrier.

\begin{tabular}{|c|c|c|c|}
\hline Question item & Alternatives & Number $=$ RQR x 100/DQ & Percent \\
\hline \multirow{3}{*}{ 1. Mode of living } & Active & 465 & $62.6 \%$ \\
\hline & Inactive (sedentary) & 278 & $37.4 \%$ \\
\hline & Total & 743 & $100 \%$ \\
\hline \multirow[t]{3}{*}{ Question item } & Alternatives & Number & Percent \\
\hline & High (daily) & 171 & $23 \%$ \\
\hline & Medium (3 to 5 days) & 193 & $25.9 \%$ \\
\hline \multirow[t]{3}{*}{ 2. How would you describe your participation in regular physical exercises? } & Low (below 3days) & 131 & $17.6 \%$ \\
\hline & Very low (sedentary) & 248 & $33.5 \%$ \\
\hline & Total & 743 & $100 \%$ \\
\hline
\end{tabular}

As shown in table 1. Description about attitudinal barrier (Q item 1 and 2) $465(62.6 \%)$ and 248 (33.5\%) respondents replied the community leads sedentary life (low exercise) respectively therefore the researchers conclude that the stated factors were showing directly attitudinal problem

Table 2. Description about time barrier.

\begin{tabular}{llll}
\hline Question item & Alternatives & Number=RQR x 100/DQ & Percent \\
\hline & Very likely $=3$ & 92 & $12.38 \%$ \\
& Likely $=2$ & 122 & $16.42 \%$ \\
3. All day is so busy now; I just don't think I can make the time to include & Somewhat likely $=1$ & 199 & $26.78 \%$ \\
physical activity in my regular schedule. & Unlikely $=0$ & 330 & $44.41 \%$ \\
& Total & 743 & $100 \%$ \\
\hline
\end{tabular}

As shown in table 2. Description about time barrier (Q item 3) 330 (44.41\%) the significant respondents were said "lack of enough time is not the major hindering barrier for engaging in physical exercise" 
Table 3. Description about social barriers.

\begin{tabular}{|c|c|c|c|}
\hline Question item & Alternatives & Number & Percent \\
\hline \multirow{5}{*}{$\begin{array}{l}\text { 4. None of my family members or friends likes to do any activity, so I don't have } \\
\text { chance to doing physical exercise }\end{array}$} & Very likely $=3$ & 211 & $28.40 \%$ \\
\hline & Likely $=2$ & 88 & $11.84 \%$ \\
\hline & Somewhat likely $=1$ & 124 & $16.69 \%$ \\
\hline & Unlikely $=0$ & 320 & $43.07 \%$ \\
\hline & Total & 743 & $100 \%$ \\
\hline
\end{tabular}

As shown in table 3. Description about social barriers (Q item 4) 320 (43.07\%) of the unlikely respondents indicating that," my family and friends participated in physical exercise this implies social barrier is not the major hindering factors.

Table 4. Description about fear of injury.

\begin{tabular}{|c|c|c|c|}
\hline Question item & Alternatives & Number & Percent \\
\hline \multirow{5}{*}{ 5. Physical Exercise can be risky } & Very likely $=3$ & 36 & $4.85 \%$ \\
\hline & Likely $=2$ & 55 & $7.40 \%$ \\
\hline & Somewhat likely $=1$ & 111 & $14.94 \%$ \\
\hline & Unlikely $=0$ & 541 & $72.81 \%$ \\
\hline & Total & 743 & $100 \%$ \\
\hline Question item & Alternatives & Number & Percent \\
\hline \multirow{4}{*}{ 6. I know too many people who have hurt themselves by overdoing physical exercise. } & Likely $=2$ & 59 & $7.94 \%$ \\
\hline & Somewhat likely $=1$ & 101 & $13.59 \%$ \\
\hline & Unlikely $=0$ & 532 & $71.60 \%$ \\
\hline & Total & 743 & $100 \%$ \\
\hline
\end{tabular}

As shown in table 4. Description about fear of injury (Q item 5 and 6) $541(72.81 \%)$ and $532(71.60 \%)$ the unlikely respondents said that," physical exercise is not harmful" because everybody were control the load and engage in physical exercise by the help of experts.

Table 5. Description about lack of resource.

\begin{tabular}{|c|c|c|c|}
\hline Question item & Alternatives & Number & Percent \\
\hline \multirow{5}{*}{ 7. How would you describe sport related services in the town? } & Very likely $=3$ & 88 & $11.84 \%$ \\
\hline & Likely $=2$ & 211 & $28.39 \%$ \\
\hline & Somewhat likely $=1$ & 268 & $36.03 \%$ \\
\hline & Unlikely $=0$ & 176 & $23.68 \%$ \\
\hline & Total & 743 & $100 \%$ \\
\hline Question item & Alternatives & Number & Percent \\
\hline \multirow{4}{*}{ 8. I don't have access to jogging trails, swimming pools, bike paths, etc. } & Likely $=2$ & 163 & $21.99 \%$ \\
\hline & Somewhat likely $=1$ & 148 & $19.97 \%$ \\
\hline & Unlikely $=0$ & 130 & $17.51 \%$ \\
\hline & Total & 743 & $100 \%$ \\
\hline \multirow[t]{3}{*}{ Question item } & Alternatives & Number & Percent \\
\hline & Very likely $=3$ & 227 & $30.53 \%$ \\
\hline & Likely $=2$ & 167 & $22.43 \%$ \\
\hline \multirow{2}{*}{ 9. It just too expensive. You have to take a class or join a club or buy the right equipment. } & Unlikely $=0$ & 231 & $31.11 \%$ \\
\hline & Total & 743 & $100 \%$ \\
\hline \multirow[t]{2}{*}{ Question item } & Alternatives & Number & Percent \\
\hline & Very likely $=3$ & 405 & $54.55 \%$ \\
\hline \multirow{4}{*}{$\begin{array}{l}\text { 10. If we had exercise facilities and showers at school or at work, then I would be more likely } \\
\text { to exercise. }\end{array}$} & Likely $=2$ & 138 & $18.52 \%$ \\
\hline & Somewhat likely $=1$ & 70 & $9.40 \%$ \\
\hline & Unlikely $=0$ & 130 & $17.51 \%$ \\
\hline & Total & 743 & $100 \%$ \\
\hline
\end{tabular}

As shown in table 5. Description about fear of injury (Q item 7, 8, 9 and 10) implies that the significant respondents respond $268(36.03 \%), 302(40.52 \%), 231(31.11 \%), 405(54.55 \%)$ respectively of the sport related services in Debre Birhan and Showa Robit town is poor, the cost of materials too much expense, resource at school and work place so scarce which leads the stated factors are the major barrier. 
Table 6. Description about lack of interest.

\begin{tabular}{|c|c|c|c|}
\hline Question item & Alternatives & Number & Percent \\
\hline \multirow{5}{*}{ 11.I ' $\mathrm{m}$ just too tired after school or work to get any exercise } & Very likely & 222 & $29.81 \%$ \\
\hline & Likely & 144 & $19.39 \%$ \\
\hline & Somewhat likely & 187 & $25.18 \%$ \\
\hline & Unlikely & 190 & $25.53 \%$ \\
\hline & Total & 743 & $100 \%$ \\
\hline \multirow[t]{3}{*}{ Question item } & Alternatives & Number & Percent \\
\hline & Very likely & 309 & $41.53 \%$ \\
\hline & Likely & 191 & $25.75 \%$ \\
\hline \multirow[t]{3}{*}{ 12.I have been thinking about getting more exercise, but I just can't seem to get started } & Somewhat likely & 126 & $16.93 \%$ \\
\hline & Unlikely & 117 & $15.77 \%$ \\
\hline & Total & 743 & $100 \%$ \\
\hline \multirow[t]{3}{*}{ Question item } & Alternatives & Number & Percent \\
\hline & Very likely & 223 & $30.01 \%$ \\
\hline & Likely & 143 & $19.24 \%$ \\
\hline \multirow[t]{3}{*}{ 13. It is easier for me to find excuse not to exercise than to go out to do something. } & Somewhat likely & 97 & $13.02 \%$ \\
\hline & Unlikely & 281 & $37.77 \%$ \\
\hline & Total & 743 & $100 \%$ \\
\hline \multirow[t]{3}{*}{ Question item } & Alternatives & Number & Percent \\
\hline & Very likely & 182 & $24.45 \%$ \\
\hline & Likely & 135 & $18.23 \%$ \\
\hline \multirow[t]{3}{*}{ 14. I'm too tired during the week and I need the weekend to catch up on my rest. } & Somewhat likely & 142 & $19.10 \%$ \\
\hline & Unlikely & 284 & $38.20 \%$ \\
\hline & Total & 743 & $100 \%$ \\
\hline \multirow[t]{3}{*}{ Question item } & Alternatives & Number & Percent \\
\hline & Very likely & 45 & $6.05 \%$ \\
\hline & Likely & 117 & $15.77 \%$ \\
\hline \multirow[t]{3}{*}{ 15. I want to get more exercise, but I just can't seem to make myself stick to anything. } & Somewhat likely & 159 & $21.30 \%$ \\
\hline & Unlikely & 402 & $54.12 \%$ \\
\hline & Total & 743 & $100 \%$ \\
\hline
\end{tabular}

As shown in table 6. Description about lack of interest (Q item 11, 12, 13, 14 and 15) 222 (29.81\%), 309 (41.53\%), very likely respectively $281(37.77 \%), 284(38.20 \%), 402(54.12 \%)$ unlikely respectively the respondents respond like that of tiredness, risky, do not going out from the door they think to work but they don't work practically, which is supporting lack of interest this implies the researchers can conclude most of the respondents said is the cause of abstains from physical exercise in Debre Birhan and Showa Robit towns.

Table 7. Description about lack of skill.

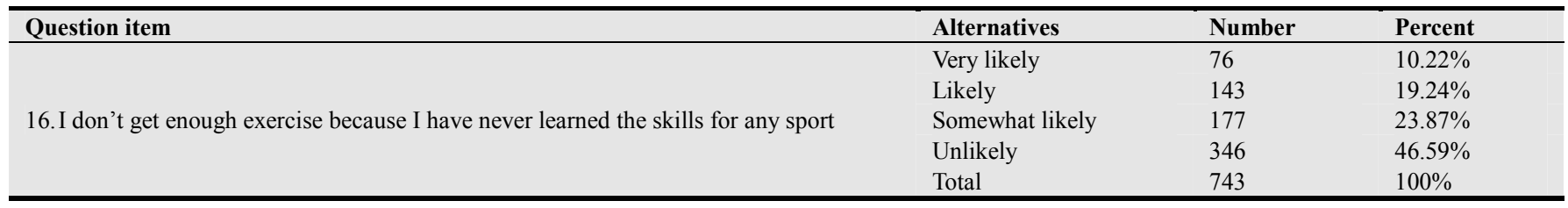

As shown in table 7. Description about lack of skill (Q item 16) 346 (46.59\%) respond unlikely because they want to learn any sport skill in Debre Birhan and Showa Robit town most of the significant respondents assuring wants to learn skill which implies skill gap is the major barrier.

Table 8. Description about barrier of health condition.

\begin{tabular}{|c|c|c|c|}
\hline Question item & Alternatives & Number & Percent \\
\hline \multirow{3}{*}{ 17. Do you check your over- all health condition periodically? } & A. Yes & 155 & 20.83 \\
\hline & B. No & 588 & 79.16 \\
\hline & Total & 743 & $100 \%$ \\
\hline
\end{tabular}

As shown in table 8. Description about barrier of health condition (Q item 17), do you check your over- all health condition periodically? $588(79.16 \%)$ respondents respond no that means the majority of the people haven't the trends of periodical checkup this implies the health status of the community indicates in Debre Birhan and Showa Robit towns facing as hindering barrier.

\section{Conclusion and Recommendation}

\subsection{Conclusion}

On the basis of the above result and discussion the researchers were concluded: The significant respondents replied that time barrier, social barrier and fear of injury were 
not the major hindering barrier engaging with physical exercise whereas the majority of the respondents in Debre Berhan and Showa Robit town most of the significant respondents replied unlikely response because they didn't start physical exercise due to of attitudinal barrier which are checked by international standard like likert scale, interview or people's opinion and literature based evidence associated to certain variables like poor lifestyle and environment (tobacco utility, weather condition, poor nutrition, leading poor sedentary life and poor hygiene), shortage of facilities and equipment's, lacking interest, missing of required skill comparing with active individuals, lack of self-confidence and lacking periodical checkup about entire health condition associated to NCDs were the significant bottleneck barrier engaging with regular physical exercise program.

\subsection{Recommendation}

On the basis of the above results, discussion and conclusion the following recommendation were providing for proper implementation of adapting physical activity with regards to:

prepared creation forum within the community jointly by the help of professionals because attitudinal barriers are the major problem, therefore: The Ministry of education, the Federal Sport commission, the concerned Sport federations, all Governmental service delivery organization, nongovernmental organization, private sectors highly responsible for the effectiveness of physical exercise at community level.

All of these are concerning professional training like:

- Provide sound education by different medium

- Provide short and long term training about the benefit of physical exercise as a mainstream.

- Provide seminar and workshop at local and national level for celebrating annual sport week

- The government mainstream the sport policy

- Revised sport science curriculum by incorporating proper credit for physical education program related to NCDs (like cardio respiratory cases) in:

- Primary and

- Secondary school level

- Trained high level experts beyond usual way

- Allocate proper annual budget for physical activity program

All stake holders responsible for access and available different sport facilities and equipment's especially government and private sectors in the case of building any infrastructures with regards to build: New villages, schools, health centers any construction projecting, consider the sport activities in our zone and town at particular. Government and private institutions think advance and build gymnasium for providing appropriate health service for employees or community at large

About community health condition for this professionals (various physicians) like medical specialists, physiotherapists and fitness instructors working jointly work about; contradictions, prescribed exercise and dosage of exercise: To enhance cardio respiratory endurance/cardiovascular fitness and to reduce over stress, anxiety and depression

About means to involve in physical exercise associated with fitness components: Aerobic (muscle, respiratory and cardiovascular endurance) exercise, anaerobic (muscle endurance and speed endurance), warming up exercise (dynamic stretching) flexibility of mobilization exercise, Strength exercise (muscle- power strength) employed weight lifting and resistance exercise, types of massage as exercise for readiness:

- Sport massage

- Recreational massage

- Rehabilitation massage is valuable with regards to means of rebuilds for various cases in the human body

\section{Acknowledgement}

First Doctor Temesgn Tebebu Mekonnen (PhD) his valuable encouragement, guidance and counseling from the beginning stage of proposal formulation as senior research staff, Second we the researchers acknowledge Debre Birhan University because encouraging and supporting through motivation and finance. Third we want to acknowledge responding participants. Lastly but not least as a principal researcher acknowledging all our staffs and co researchers especially (Mr. Abiot Kassaw, Mr. Belay Lema and Mr. Birhanu Anjte from the beginning of the issue supporting to their continuous commitment with the whole research organ accomplishment.

\section{References}

[1] (Background document prepared for WHO workshop on physical activity and public Health 24-27october 2005 Beijing peoples of Republic of China).

[2] Booth ML, Owen N, Bauman A, Gore CJ. Physical activity preferences, preferred sources of assistance and perceived barriers to increased activity among physically inactive Australians. Pre Med. 1997; 26: 131-137.

[3] Calfas KJ, Long BJ, Sallis JF, Wooten WJ, Pratt M, Patrick K. A controlled trial of physician counseling to promote the adoption of physical activity. Prev Med. 1996; 25: 255-233.

[4] Cardinal BJ. Behavioral and biometric comparisons of the preparation, action, and maintenance stages for exercise. Research, Theory, and Practice. 1995; 11: 36-43.

[5] (Christine Hancock, Why invest in physical activities 2011).

[6] Dishman RK. Advances in Exercise and Adherence. Champaign, IL: Human Kinetics Books, 1994.

[7] Franis KT. Status of the year 2000 health goals for physical activity and fitness. Phys Ther. 1999; 79: 404-14.

[8] Marcus BH, Bock BC, Pinto BM, Forsyth LH, Roberts LH, Traficante RM. Efficacy of an individualized motivationally tailored physical activity intervention. Ann Beh Med. 1998; 20: 174-180. 
[9] Morrow JR., Jackson AW, Bazzarre TL, Milan D, Blair SN. A one- year follow-up to physical activity and health. A report of the Surgeon General. Am J Prev Med. 1999; 17: 24-3.

[10] Prochaska JO, DiClemente CC. Stages and processes of selfchange of smoking: toward an integrative model of change. $\mathrm{J}$ Consulting Clinical Psychol. 1983; 51: 390-395.

[11] Reibe D. change for the better: understanding the process of change to help ease clients into a healthy lifestyle. American Fitness. 1997; 15: 61-64.

[12] Taylor W, Baranowski T, Young D. Physical activity interventions in low-income ethnic minority and populations with disability. Am J Prev Med. 1998; 15: 334-343.
[13] Sallis JF, Haskell WL, Fortmann SP, Vranizan KM, Taylor CB, Solomon DS. Predictors of adoption and maintenance of physical activity in a community sample. Prev Med. 1986; 15: 331-334.

[14] U.S. Department of Health and Human Services. Physical activity and health: A report of the Surgeon General. Atlanta, GA: U.S. Department of Health and Human Services, Centers for Disease Control and Prevention; 1996.

[15] Theoretical models and the adoption of physical activity (Tara Spruce Stimson). 